is used to assess organ dysfunction, including liver. According to this scale, the main criterion for liver damage is an increase in alanine aminotransferase (ALT) activity over 50\% of baseline. Also, the enzymatic activity of ALT may be increased in newborns with parenteral nutrition.

Objectives The purpose of the study: to study the clinical and laboratory, instrumental and pathomorphological features of septic hepatitis in newborns.

Methods An analysis of 32 inpatient maps of children (of which 29 - (90.6\%) premature in gestation 27 - 34 weeks) with septic hepatitis. The only diagnostic criterion was considered to be a combination of the above components with systemic inflammatory response syndrome (SIRS - systemic inflammatory response syndrome).

Results Hepatomegaly was detected in 25 (78\%) patients. Hemorrhagic syndrome was detected in 22 (68.7\%) infants, most often in the form of pulmonary or gastrointestinal bleeding. Changes in hemostasis in patients were accompanied by an increase in activated. Splenomegaly was detected in 3 (9.4\%) children as a result of hyperplasia of the reticulogistiocytic tissue of the organ in response to sepsis and hepatitis. Conjugated hyperbilirubinemia was detected in all 32 patients (100\% of cases). Decreased prothrombin index was observed in 25 children (78\%), and increased levels of alanine aminotransferase and aspartate aminotransferase (ALT, AST) in 23 (72\%). Hypoproteinemia was observed in 12 children $(37.5 \%)$. Ultrasound in septic hepatitis in 28 (87.5\%) patients was characterized mainly by changes in the liver parenchyma and its vascular system, sometimes in combination with biliary tract lesions in the form of thickening of the gallbladder walls and heterogeneity of its contents.

Conclusions Clinical symptoms of septic hepatitis are accompanied by jaundice, in most children hepatomegaly and hemorrhagic syndrome. The main laboratory criteria for septic hepatitis were: conjugated hyperbilirubinemia (100\%), decreased prothrombin index $(78 \%)$ and increased levels of ALT and AST (72\%). The most unfavorable prognosis was observed as a result of penetration of the pathogen into the liver through the umbilical vein. In these cases, morphologically formed necrotic hepatitis with total damage to all parts of the hepatobiliary system. The clinical symptoms of the disease were characterized by severe hepatic insufficiency with impaired basic liver function and very high mortality.

\section{CHALLENGES IN THE MANAGEMENT OF EXTENSIVE APLASIA CUTIS CONGENITA}

Alvin Ngeow, Cheryl Hui, Mark Koh, Darryl Chew, Yee Siang Ong, Woei Bing Poon. Singapore

\subsection{6/bmjpo-2021-RCPCH.115}

Background Aplasia cutis congenita (ACC) is a rare group of congenital disorders characterised by focal or widespread absence of skin, predominantly affecting the scalp, although other areas of involvement have been reported. The exact pathogenesis is unknown, although several theories have been proposed including neural tube defect, vascular compromise from placental insufficiency, intra-uterine infections, genetic mutations, teratogens, and ischemic or thrombotic events related to fetus papyraceus. ACC patients with large scalp defects, often associated with skull defects and/or exposed dura and superior sagittal sinus have reportedly suffered morbidity or demised from catastrophic haemorrhage from the sagittal sinus, and/or suffered meningitis. Diagnosis is mostly clinical. There is no consensus for the management of ACC given the rarity and variability in terms of the clinical presentation (site) and extent of ACC. Management approaches include conservative treatment, which involves dressing of the aplastic skin until complete re-epithelialisation, and surgical management, which include excision and closure, skin grafting, local flaps and tissue expansion.

Objectives This report describes the treatment of an extensive case of ACC.

Methods The child was managed by a multidisciplinary team comprising the neonatologist, paediatric dermatologist, plastic surgeon, hand surgeon and geneticists.

Results The areas of involvement included most of the scalp and posterior neck, with sparing of the forehead, anterior chest and abdomen, with small portions of the back. Small areas of ACC at joint creases of lower limbs and bilateral upper limbs and bilateral groin regions were also noted. In all, 33.5\% body surface area was involved. Both conservative and surgical approaches were considered. On top of the inherent risks associated with conservative management of ACC, such as wound infection, electrolyte abnormalities, and delayed wound healing, this baby was at risk of potentially catastrophic haemorrhage and meningitis due to the large scalp defect. As such, coverage for the scalp was prioritised. Options for skin cover included allograft versus autograft; latter was preferred due to reduced risk of rejection. This approach was limited by the availability of donor sites due to extensive ACC and risks of donor site morbidity. Cultured epithelial autograft (CEA) was preferred but could only be ready after three weeks of tissue culture. As a bridging measure, artificial dermal matrix (ADM) in the form of Integra (R) was applied to the scalp, and other areas, prior to application of the CEA at about 3 weeks of life. The baby was essentially planned for a 2-stage approach to provide urgent skin cover. The baby also received empiric antibiotic coverage and regular wound dressing with topical antibiotics.

Conclusions Aplasia cutis congenita is a rare and potentially life threatening congenital similar to a burns patient, but can suffer from catastrophic morbidity related to exposed sagittal sinus in cases where scalp involvement is extensive. In such cases, we propose prompt surgical coverage of the scalp. We report treatment of a case of extensive ACC involving most of the scalp, using a 2-stage approach, which first entails the application of Artificial Dermal Matrix (ADM), followed by the application of Culture Epithelial Autograft (CEA) when the graft is ready.

\section{PHENOMENOLOGY OF PEDIATRIC MOVEMENT DISORDERS: FIRST STEP FOR DIAGNOSIS}

Yeemon Wint Aung, Aye Mu San. Myanmar

\subsection{6/bmjpo-2021-RCPCH.116}

Background Movement disorders are broad group of acute and chronic neurological conditions in children and it has been increasingly recognized worldwide because of disturbance of activities of daily living and quality of life. Because of diagnostic and therapeutic challenges for movement disorders, phenomenology plays the key role in diagnosis of specific 
movement disorders and also helps in identifying the underlying causes.

Objectives The objectives of study are to classify the types of movement disorders, to identify the underlying cause and to assess the short term outcomes of children with movement disorders.

Methods Cross sectional descriptive study was conducted in all children with movement disorders attending hospital during one year period. Phenomenology of movement disorders were classified by visual pattern recognition or recorded home based videos using smartphone. Relevant clinical examination and laboratory investigations including EEG and imaging were done. Underlying causes and short term outcomes were found out.

Results Of 102 patients, types of movement disorders were dystonia $(n=39)$ followed by chorea and athetosis $(n=32)$, tics $(n=12)$, tremor $(n=8)$, stereotypes $(n=5)$, myoclonus $(n=4)$ and bradykinesia $(n=2)$. Sixty per cent of the cases were older than 5 years of age. Nearly $60 \%$ of children were male. Age of onset of movement disorders more than 5 years of age is $46 \%$ of children. The median duration of abnormal movements were 345 days. The main causes of dystonia were birth asphyxia $(n=13)$ and infection $(n=9)$. The major causes for chorea and athetosis were infection $(n=12)$ and kernicterus $(n=12)$. Common cause of infections were encephalitis Among study population, 74\% of dystonic children, $53 \%$ chorea and athetosis, $25 \%$ of tics, $62.5 \%$ of tremor, $50 \%$ of myoclonus and bradykinesia were improved. Twenty-six percent of dystonia, 31\% of chorea and athetosis, $75 \%$ of tics, $37.5 \%$ children with tremor and $50 \%$ of myoclonus had persistent symptoms. Only $16 \%$ of chorea and athetosis and $50 \%$ of bradykinesia had completely recovered. Dystonia with underlying CNS infection, chorea and athetosis due to Syndeham's Chorea and Wilson disease, tics, tremor due to drug-induced cases, autoimmune encephalitis and paroxysmal tremor and myoclonus of infectious encephalitis and autoimmune encephalitis were improved. Dystonia and choreo-athetosis due to cerebral palsy, all cases of sterotypes, tremor due to ALL with CNS metastasis and neurometabolic disease were persisted.

Conclusions Phenomenology plays the key role in diagnosis of specific movement disorders and also helps in identifying the underlying causes. Understanding to phenomenology and clinical profile of movement disorder in children is very crucial for correct diagnosis and management. This study pointed out that childhood movement disorders are common and most of the common movement disorders are preventable or treatable cause such as neuroinfections, immune aetiologies, hypoxic injury at birth, kernicterus and drug induced movement orders. Because of diverse aetiologies pose a diagnostic challenge to pediatrician, emphasis on phenomenology plays a role. Focusing on those factors, diagnosis approach of pediatric movement disorders can be simplified.

\section{THE POSITIVE THINKING PROJECT - CAN WE IMPROVE TRAINEE MORALE?}

Alexandra Damazer, Lesley Clarkson, Mary Barraclough. UK

10.1136/bmjpo-2021-RCPCH.117

Background Doctor's health and wellbeing are critical to the quality of care they provide, both to their patients and to themselves. ${ }^{1}$ Thirty percent of doctors in the UK are not satisfied with their day to day work and in 2019 eighteen percent of doctors in training reported having to take time off work due to stress. ${ }^{2}$ It is also acknowledged that doctors in training are more positive about their experiences when working in a supportive workplace. $^{2}$

GMC National Training Survey results for the last three years show that there has been a fall in trainee satisfaction with feedback received in the Paediatric Department at Hull University Teaching Hospitals. ${ }^{3}$

Objectives The Positive Thinking Project aimed to improve trainee feedback from Consultants, through a weekly positive feedback statement and a point for development.

Secondary objective was to demonstrate the role of trainee feedback in improving trainee morale within a paediatric department.

Methods A proforma with name, grade and photograph of all trainee doctors and advanced nurse practitioners was circulated to the Consultant of the Week (COW) every week. The COW was asked to provide a positive feedback statement and point for development for each trainee who had been at work in the department during their COW week. The completed proformas were collated confidentially, and trainees received their personalised feedback via email every three months.

Trainees were then asked to give feedback on the positive thinking project, either verbally or by email. Feedback was collated over a 12 month period and anonymised. Analysis was carried out using an inductive approach and open coding. Results Seven codes were identified, with three overriding themes. Data saturation was reached.

\begin{tabular}{lll} 
Abstract 217 Table 1 & & \\
\hline Codes & Theme & Examples \\
\hline Gratitude/Thanks & Positivity & $\begin{array}{l}\text { 'really does brighten my } \\
\text { day' }\end{array}$ \\
$\begin{array}{l}\text { Positive emotions } \\
\text { Project positivity }\end{array}$ & 'thank you so much' \\
$\begin{array}{l}\text { Receiving feedback } \\
\text { Personal/career }\end{array}$ & 'it is lovely to receive.' \\
development & Development & 'very positive feedback' \\
Feedback as evidence & 'constructive' \\
Element of Surprise & 'evidence in our portfolio' \\
& Surprise & 'very unexpected' \\
\hline
\end{tabular}

Conclusions Feeling valued is a cornerstone of improved morale and motivation within the NHS. ${ }^{4}$ The Positive Thinking Project aimed to focus on the provision of regular, positive feedback for trainees from Paediatric Consultants, praising what has been done well and giving an area for development. We have demonstrated that trainees feel feedback has been positive, constructive and helpful for development and that this project gave trainees feedback that they do not feel they would have otherwise received.

\section{REFERENCES}

1. General Medical Council. Caring for Doctors, Caring for Patients. Published 2019.

2. NHS Health Education England. Junior Doctor Morale Understanding Best Practice Working Environments. Published 2017.

3. General Medical Council. National Training Survey. 2019, 2018, 2017.

4. King's Fund. Counting the Smiles Morale and Motivation in the NHS. Published 2002. 